\title{
Multi-Objective Resource Allocation for LTE/LTE-A Femtocell/HeNB Networks using Ant Colony Optimization
}

\author{
Ying Loong Lee • Jonathan Loo · Teong \\ Chee Chuah • Ayman A. El-Saleh
}

Received: date / Accepted: date

\begin{abstract}
Existing femtocell resource allocation schemes for Long Term Evolution (LTE) or LTE-Advanced femtocell networks do not jointly achieve efficient resource utilization, fairness guarantee, interference mitigation and reduced complexity in a satisfactory manner. In this paper, a multi-objective resource allocation scheme is proposed to achieve these desired features simultaneously. We first formulate three objective functions to respectively maximize resource utilization efficiency, guarantee a high degree of fairness and minimize interference. A weighted sum approach is then used to combine these objective functions to form a single multi-objective optimization problem. An ant colony optimization algorithm is employed to find the Pareto-optimal solution to this problem. Simulation results demonstrate that the proposed scheme performs jointly well in all aspects, namely resource utilization, fairness and interference mitigation. Additionally, it maintains satisfactory performance in the handover process and has a reasonably low complexity compared to the existing schemes.
\end{abstract}

Keywords LTE · LTE-A · Femtocell · Resource Allocation · Multi-Objective Optimization · Ant Colony Optimization

\section{Introduction}

Femtocell technologies have received immense interest from both academia and industry due to their potential in achieving the Long Term Evolution

Y. L. Lee · T. C. Chuah · A. A. El-Saleh

Faculty of Engineering, Multimedia University, Jalan Multimedia, 63100 Cyberjaya, Selangor, Malaysia

E-mail: lee.ying.loong12@student.mmu.edu.my, \{tcchuah, ayman.elsaleh\}@mmu.edu.my

J. Loo

School of Science and Technology, Middlesex University, London NW4 4BT, United Kingdom

E-mail: J.Loo@mdx.ac.uk 
(LTE) or LTE-Advanced (LTE-A) specifications. In LTE/LTE-A, femtocell base stations, also known as Home evolved NodeBs (HeNBs), are intended to be deployed indoor to fill the coverage holes caused by weak signals from macrocell base stations, also known as evolved NodeBs (eNBs). The low power and low cost features of femtocells make them an attractive solution to improve indoor coverage [1]. As the data in the femtocell backhaul can be carried via indoor broadband wirelines, traffic congestion at the macrocell can also be reduced [1]. Although femtocells look promising, several challenges in resource allocation remain to be addressed.

Interference is one of the main challenges of resource allocation in LTE/LTEA femtocell networks. In an LTE/LTE-A femtocell network, co-tier interference occurs when HeNBs mutually interfere with each other and cross-tier interference occurs when HeNBs interfere with the eNB [1]. In general, there are two interference mitigation approaches: shared and split spectrum. The shared spectrum approach allows both macrocell and femtocell to utilize the whole channel bandwidth without interfering each other. However, the shared spectrum approach may introduce security, scalability and limited backhaul bandwidth issues [2] and cause femtocells to lose the fundamental advantages of resource reuse [3]. On the other hand, the split spectrum approach avoids cross-tier interference entirely by dividing the system bandwidth to macrocell and femtocell, thus simplifying its resource allocation mechanism into only mitigating co-tier interference.

As the split spectrum approach is simpler than the shared spectrum approach, a number of efficient resource allocation schemes have been developed for this approach [4-12]. In [4], a random hashing function is used to avoid allocating the same resources to interfering femtocells. However, its design does not consider satisfying the resource demands imposed by the HeNBs. Thus, it does not achieve globally fair resource allocation [13] and quality of service (QoS) may not be guaranteed. In [5], [6] and [7], a central entity is used to identify interfering femtocells and avoid resource reuse among them. However, resource utilization maximization is not considered in [5]. Furthermore, the complexity of the schemes proposed in [6] and [7] is prohibitively high, especially for large femtocell networks, because the central entity needs to perform resource allocation among the data flows associated with all the femtocells. In [8] and [9], transmission power control schemes are used to reduce co-tier interference. However, global fairness is not addressed in [8] and resource utilization maximization is not considered in [9]. Graph coloring techniques are used in [10] and [11] to avoid co-tier interference and to maximize throughput, but global fairness is not considered because the techniques do not take into account the resource demands of the HeNBs. In [12], a clustering method is used to group interfering femtocells and avoid resource reuse within the group. However, the computational load is shifted to the HeNB that serves as the cluster head. Since the HeNBs are generally not equipped with high processing capabilities like those of the eNB, the computational load could still be excessive for an HeNB. 
As emphasized in [13], resource allocation in femtocell networks is a multiobjective problem which should jointly consider interference mitigation, efficient resource utilization, global fairness and complexity. In the current work, a multi-objective resource allocation scheme with reasonable complexity is proposed for LTE/LTE-A femtocell networks. We consider femtocells that operate in the open access mode as they can provide better system-level performance compared to closed access femtocells $[14,15]$. The current work only focuses on mitigating co-tier interference and it is assumed that cross-tier interference has been alleviated by the split spectrum approach. The main contributions of this paper are summarized as follows: 1) Three resource allocation objective functions are formulated with one for resource utilization maximization, second for fairness maximization and the third for interference minimization; 2) The aforementioned three optimization problems are combined into a single optimization problem using a weighted sum approach and a weight setting method is proposed; 3) A resource allocation algorithm based on ant colony optimization (ACO), which is based on the behavior of ants in search of food, is devised to solve the optimization problem; and 4) Performance studies of the proposed algorithm and the existing prominent schemes in terms of throughput, fairness, resource utilization and complexity.

The remainder of this paper is organized as follows. Section 2 describes the LTE/LTE-A system model, the multi-objective resource allocation problem formulation and the proposed multi-objective resource allocation scheme. Performance results are presented in Section 3. Section 4 provides the conclusion.

\section{Proposed Multi-Objective Resource Allocation Scheme}

In this section, the system model of an LTE/LTE-A femtocell network is described followed by the formulation of the resource allocation optimization problem and the proposed solution algorithm.

\subsection{System Model}

We assume that the femtocells deployed in each indoor building form a femtocell network. It is noteworthy that interference may occur between adjacent indoor buildings where the femtocells are deployed. Though the interference may be negligible, it can be avoided by dividing the available bandwidth into several portions and reusing them among the buildings. However, co-tier interference still exists within the femtocell network in each building and its mitigation is the focus of the current work. For simplicity, we only consider one femtocell network and adopt the system model in [7] which consists of an HMS and several HeNBs and FUEs [16]. In this model, the HMS performs resource allocation among the HeNBs within the femtocell network. Before this, the HMS needs to first collect the interference and resource demand information from all the connected HeNBs. 
In LTE/LTE-A systems, the channel bandwidth is divided into a number of subchannels each of $180 \mathrm{kHz}$. Each subchannel is further divided in the time domain into a number of physical resource blocks (PRBs) [17] each with a duration of $0.5 \mathrm{~ms}$. These PRBs are scheduled and allocated to the user equipment (UE) every one time transmission interval (TTI) of $1 \mathrm{~ms}$. In this paper, we focus on downlink subchannel allocation among HeNBs. Let $\mathcal{H}, \mathcal{K}$, $\mathcal{U}_{h}$ and $\mathcal{C}_{u}$ denote the sets of HeNBs, subchannels, FUEs of HeNB $h$ and data flows of HeNB $u$, respectively. Note that |.| refers to the cardinality of the set. Without loss of generality, we assume that 1) the bandwidth of each PRB is less than the coherence bandwidth of the channel, i.e., each PRB experiences flat fading; 2) The time duration of each PRB is shorter than the coherence time of the channel, i.e., each PRB experiences slow fading; and 3) the femtocell network is perfectly synchronized.

In LTE/LTE-A systems, each UE can carry a number of data flows $[18,19]$ where each data flow can be classified as either a guaranteed bit rate (GBR) flow or a non-GBR flow. A GBR flow is imposed with a minimum bit rate which serves as the minimum QoS requirement. On the other hand, non-GBR flows are not imposed with the minimum bit rate constraint and thus the number of resources to be assigned to these flows depends on the resource allocation scheme used. In this work, we assume that the minimum resource demand of each non-GBR flow is one. Let $D_{c}$ be the minimum resource demand of data flow $c$, which can be estimated as

$$
D_{c}= \begin{cases}\left\lceil\frac{R_{c}^{\text {req }}}{f_{\mathrm{PRB}} S_{\mathrm{E}}}\right\rceil & \text { if data flow } c \text { is a GBR flow } \\ 1 & \text { otherwise }\end{cases}
$$

where $R_{c}^{\text {req }}$ is the minimum bit rate of data flow $c, f_{\mathrm{PRB}}=180 \mathrm{kHz}$ and $S_{\mathrm{E}}$ is the achievable spectral efficiency in bits $/ \mathrm{s} / \mathrm{Hz}$ when a specific modulation and coding scheme (MCS) is selected for transmission. $S_{\mathrm{E}}$ can be estimated based on the wideband channel quality indicator (CQI) from the adaptive modulation and coding (AMC) module in the medium access control (MAC) layer of the LTE/LTE-A system [19]. Thus, the minimum resource demand of HeNB $h$ is the sum of the minimum resource demands of its associated data flows, i.e.,

$$
D_{u}=\sum_{c \in \mathcal{C}_{u}} D_{c}
$$

and the minimum resource demand of each HeNB $h$ is the sum of the minimum resource demands of its associated FUEs, i.e.,

$$
D_{h}=\sum_{u \in \mathcal{U}_{h}} D_{u}
$$

The interference in an LTE/LTE-A femtocell network can be modeled using an interference graph as shown in Fig. 1. In this graph, a node/vertice represents an HeNB and the solid links/edges connect nodes that interfere 


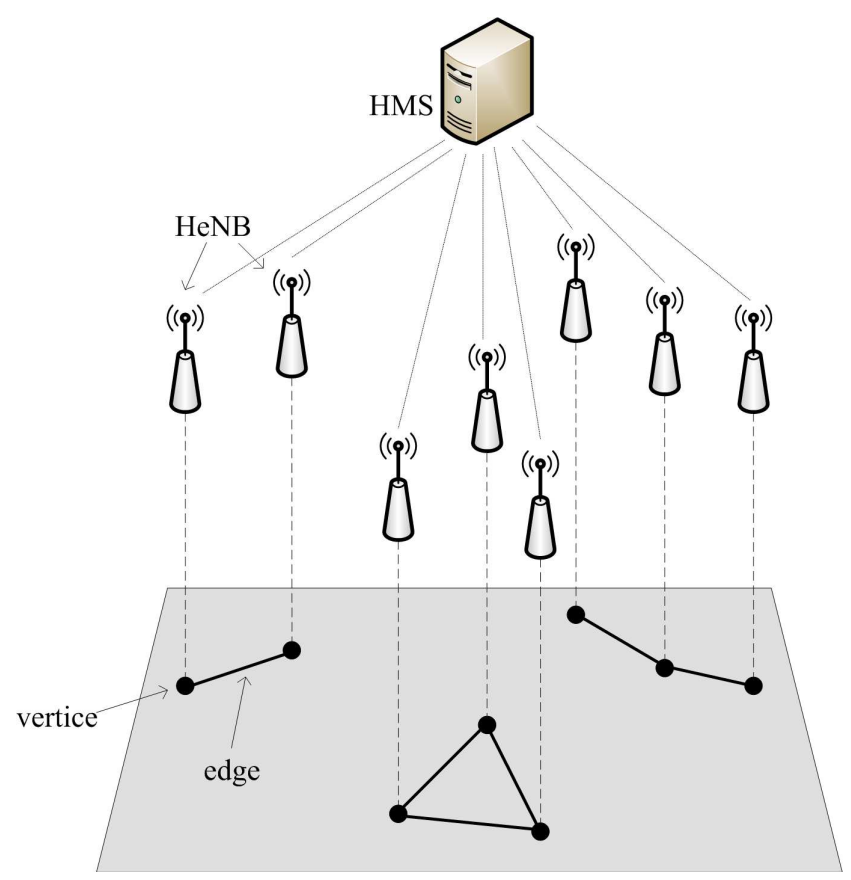

Fig. 1 A three-layer graph

with each other. Based on the interference graph, the interference relationship among the HeNBs can be described using a matrix $\mathbf{A}=\left[a_{i j}\right]_{|\mathcal{H}| \times|\mathcal{H}|}$ where

$$
a_{i j}= \begin{cases}1 & \text { if HeNB } j \text { interferes with HeNB } i \\ 0 & \text { otherwise }\end{cases}
$$

In this work, we follow the approach in [20] where each femtocell UE (FUE) periodically measures the received signal strength (RSS) of the LTE/LTE-A reference signals transmitted by all the HeNBs in the network. The measurement reports are sent back to the serving HeNB, and the interfering HeNBs can be identified from the reports by using the following inequality [20]

$$
P_{u i}^{\mathrm{ref}}(\mathrm{dB})<P_{u j}^{\mathrm{ref}}(\mathrm{dB})+T h(\mathrm{~dB})
$$

where $P_{u i}^{\text {ref }}$ and $P_{u j}^{\text {ref }}$ are the RSSs received by FUE $u$ from the serving HeNB $i$ and neighboring HeNB $j$, respectively, and $T h$ is a protection margin that takes into account the aggregate interference from the neighboring macrocells and fading effects. If (5) holds, then $a_{i j}=1$, otherwise $a_{i j}=0$. Then, an interference vector $\mathbf{a}_{h}=\left[a_{h 1}, a_{h 2}, \ldots, a_{h|\mathcal{H}|}\right]$ for HeNB $h$ can be formed. After that, each HeNB sends its own interference vector to the HMS to form matrix $\mathbf{A}=\left[\mathbf{a}_{1}, \mathbf{a}_{2}, \ldots, \mathbf{a}_{|\mathcal{H}|}\right]^{\mathrm{T}}$. 
2.2 Problem Formulation

The main objective of the current work is to maximize resource utilization efficiency and global fairness while minimizing co-tier interference. Let $\omega_{h k}$ be the assignment indicator of subchannel $k$ to $\operatorname{HeNB} h$, e.g., $\omega_{h k}=1$ if subchannel $k$ is assigned to HeNB $h$, otherwise $\omega_{h k}=0$. The objective function that maximizes the resource utilization efficiency of a femtocell network can be formulated as

$$
f_{1}=\frac{\sum_{h \in \mathcal{H}} \sum_{k \in \mathcal{K}} \omega_{h k}}{|\mathcal{H}||\mathcal{K}|}
$$

This objective function sums the number of times each resource is being reused among all the HeNBs. The denominator $|\mathcal{H}||\mathcal{K}|$ normalizes the value of $f_{1}$ within the range $[0,1]$.

To achieve fair resource allocation among the HeNBs, the amounts of resources allocated should be proportional to their resource demands. To achieve this, the score of the following function should be made as small as possible

$$
\delta(h, i)=\left|\left(\sum_{k \in \mathcal{K}} \omega_{h k}-D_{h}\right)-\left(\sum_{k \in \mathcal{K}} \omega_{i k}-D_{i}\right)\right| \quad \forall i \in \mathcal{H} \backslash\{h\}
$$

where $\delta(h, i)$ indicates the gap between the proportion of resources allocated to HeNB $h$ and that to HeNB $i$. It is noteworthy that $|x|$ in (7) denotes the absolute value of $x$. From (7), the objective function that minimizes the proportion gap can be formulated as

$$
f_{2}=\frac{\sum_{i \in \mathcal{H} \backslash\{h\}} \delta(h, i)}{\sum_{i \in \mathcal{H} \backslash\{h\}}\left(\max \left(|\mathcal{K}|-D_{h}+D_{i},|\mathcal{K}|+D_{h}-D_{i}\right)-\left|D_{h}-D_{i}\right|\right)}
$$

The denominator of $f_{2}$ is normalizes the value of $f_{2}$ within the range $[0,1]$, where the first term in the sum expression can be equivalently expressed as

$$
\max \left(|\mathcal{K}|-D_{h}+D_{i},|\mathcal{K}|+D_{h}-D_{i}\right)= \begin{cases}|\mathcal{K}|-D_{h}+D_{i} & \text { if }\left(|\mathcal{K}|-D_{h}+D_{i}\right) \\ & >\left(|\mathcal{K}|+D_{h}-D_{i}\right) \\ |\mathcal{K}|+D_{h}-D_{i} & \text { otherwise }\end{cases}
$$

It is noteworthy that fairness can be maximized by minimizing $f_{2}$.

The objective function that minimizes co-tier interference can be formulated as

$$
f_{3}=\frac{\sum_{i \in \mathcal{H}} \sum_{j \in \mathcal{H} \backslash\{i\}} a_{i j} \sum_{k \in \mathcal{K}} \omega_{i k} \omega_{j k}}{|\mathcal{K}||\mathcal{H}|(|\mathcal{H}|-1)}
$$

In (10), $f_{3}$ sums the number of times each resource is being reused among all the interfering HeNBs. The denominator term $|\mathcal{K}||\mathcal{H}|(|\mathcal{H}|-1)$ is introduced to normalize the value of $f_{3}$ within the range $[0,1]$.

It is noteworthy that the objective functions in (6), (8) and (10) contradict each other. To address these conflicts, we pursue a solution that is said to be Pareto-optimal [21], which is a tradeoff between contradicting objective 
functions, where the solution cannot be further improved without degrading other objective functions. To find the Pareto-optimal solution to the three objective functions, we employ the weighted sum approach [22] to combine these objective functions into a single-objective maximization problem as

$$
\max w_{1} f_{1}+w_{2}\left(1-f_{2}\right)+w_{3}\left(1-f_{3}\right)
$$

where $w_{1}, w_{2}$ and $w_{3}$ are the relative weights for $f_{1}, f_{2}$ and $f_{3}$, respectively. It is noteworthy that $w_{1}+w_{2}+w_{3}=1$ such that the value of the objective function in (11) is within $[0,1]$. In $(11), f_{2}$ and $f_{3}$ are subtracted from unity to form the maximization problem because originally they are intended to be minimized. The solution to (11) is Pareto-optimal if the weights are set positive-valued [23]. The main advantage of this formulation is to provide the LTE/LTE-A system the flexibility to steer the focus of (11) toward any one of the objective functions by tuning their respective weight according to the operator's preference.

As the smallest change (i.e., the step size) of each of the three objective functions is different, direct weight settings can lead to unintended optimization outcomes. Assuming that all the objective functions are equally preferred, an equal weight setting will prioritize the objective function in which its smallest change is the largest compared to others. Thus, the weight values must be assigned such that the smallest change of all the objective functions is equalized among each other.

Let $A=\sum_{i \in \mathcal{H} \backslash\{h\}}\left(\max \left(|\mathcal{K}|-D_{h}+D_{i},|\mathcal{K}|+D_{h}-D_{i}\right)-\left|D_{h}-D_{i}\right|\right)$, and $(x, y, z)$ denotes the preference vector where $x, y$ and $z$ are the non-negative preference values for $f_{1}, f_{2}$ and $f_{3}$, respectively and $x+y+z=1$. From (11), the smallest change of $f_{1}, f_{2}$ and $f_{3}$ are $\frac{1}{|\mathcal{H}||\mathcal{K}|}, \frac{1}{A}$ and $\frac{1}{|\mathcal{K}||\mathcal{H}|(|\mathcal{H}|-1)}$, respectively. To equalize these smallest changes and tune the weights according to the preference vector, the weights can be set as follows

$$
w_{1}=\frac{x q}{A(|\mathcal{H}|-1)}, \quad w_{2}=\frac{y q}{|\mathcal{K}||\mathcal{H}|(|\mathcal{H}|-1)}, \quad w_{3}=\frac{z q}{A}
$$

where $q$ is a constant which can be determined as

$$
q=\frac{A|\mathcal{K}||\mathcal{H}|(|\mathcal{H}|-1)}{x|\mathcal{H}|+y A+z|\mathcal{K}||\mathcal{H}|(|\mathcal{H}|-1)}
$$

\subsection{Proposed Algorithm}

To solve (11), a global optimization technique known as ant colony optimization (ACO) [24], which is inspired from the cooperative behavior of ant colonies in search of food, is used. The ACO algorithm has been an efficient method for solving combinatorial problems involving binary design variables such as (11). In ACO, the given optimization problem is represented as a multi-layer graph [25]. The number of layers is determined by the number of design variables in the given optimization problem. For example, the three binary design 


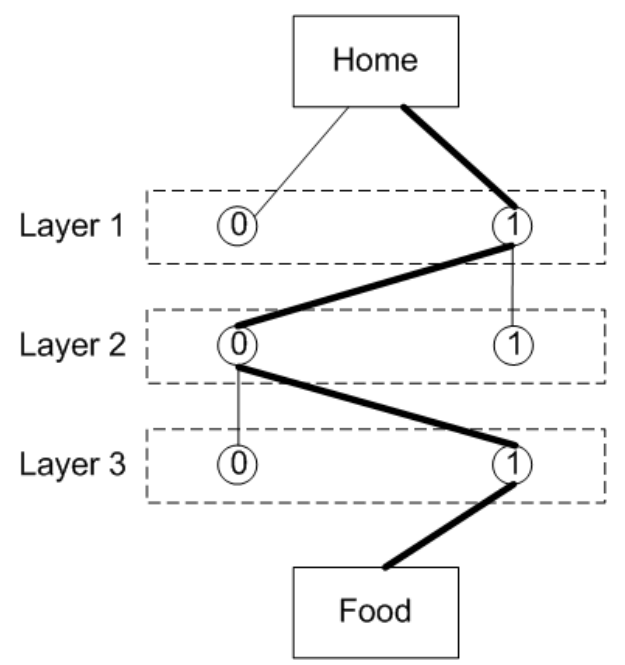

Fig. 2 A three-layer graph

variables of an optimization problem can be represented respectively in each layer of the three-layer graph depicted in Fig. 2. Since the design variables are binary-valued (i.e., either zero or one), each ant has two possible paths when traversing from one layer to another. The main idea of ACO is to employ a group of ants to travel from the home node across each layer to the food destination. The path traversed by each ant forms a candidate solution. One such path is illustrated in Fig. 2 where the bold path represents a candidate solution vector of $[1,0,1]$. The final target of ACO is to find the shortest path from the home node to the food destination. To achieve this, each ant randomly selects a path based on the pheromone trail on each path. If the pheromone trail on a path is strong, the ant is more likely to select this path. If the best path is found, the ants leave an amount of pheromones on the path to increase the probability of traversing the path again in the next food search iteration. The traversal process is repeated until a certain criterion is satisfied.

When an ant traverses from one layer to the next, the ant randomly selects a node based on the amount of pheromones deposited on the path to the node. Let $\tau_{i j}(t)$ be the pheromone trail on the path to node $j$ in layer $i$ at iteration $t$, the probability of ant $n$ selecting node $j, p_{i j}^{(n)}$ is given as [24]

$$
p_{i j}^{(n)}= \begin{cases}\frac{\tau_{i j}^{\alpha}(t)}{\sum_{m \in \mathcal{N}_{i}^{(n)}} \tau_{i j}^{\alpha}(t)} & \text { if } j \in \mathcal{N}_{i}^{(n)} \\ 0 & \text { if } j \notin \mathcal{N}_{i}^{(n)}\end{cases}
$$

where $\alpha$ is the degree of importance of the pheromones and $\mathcal{N}_{i}^{(n)}$ is the set of nodes that can be traversed to by ant $n$ in layer $i$. After all the ants have traversed from the home node to the food destination, the ants return to the 


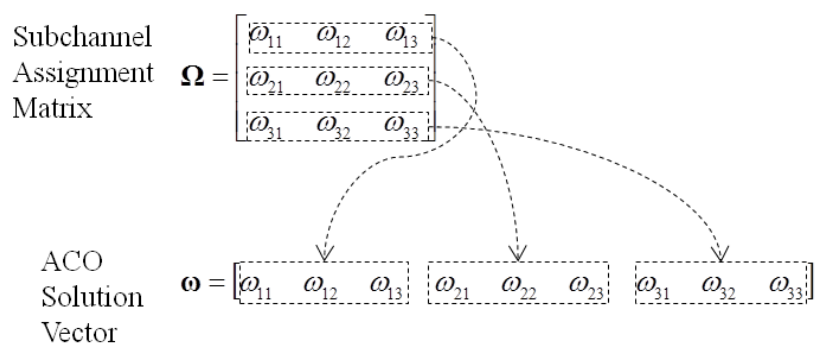

Fig. 3 Mapping between the subchannel assignment matrix and the ACO solution vector

home node and the pheromone trail of each path is updated as [24]

$$
\tau_{i j}(t+1)=(1-\rho) \tau_{i j}(t)+\sum_{n=1}^{N} \Delta \tau_{i j}^{(n)}
$$

where $\rho$ is the rate of pheromone evaporation with values in the range of $(0,1]$, $N$ is the number of ants in the colony and $\Delta \tau_{i j}^{(n)}$ is the amount of pheromones deposited by ant $n$ on path from node $i$ to node $j . \Delta \tau_{i j}^{(n)}$ is calculated as [25]

$$
\Delta \tau_{i j}^{(n)}= \begin{cases}\frac{\zeta f_{\text {best }}(t)}{f_{\text {worst }}(t)} & \text { if the path to node } j \text { in layer } i \text { is the best path } \\ 0 & \text { otherwise }\end{cases}
$$

where $f_{\text {best }}(t)$ and $f_{\text {worst }}(t)$ represent respectively the best and worst values of the objective function among the paths traversed by all the ants at iteration $t$, and $\zeta$ is a control parameter that sets the scale of the global update of the pheromone to improve the local search ability.

The aim of (11) is to optimize the subchannel assignment indicator $\omega_{h k}$ for all $h \in \mathcal{H}$ and $k \in \mathcal{K}$. Let $\Omega=\left[\omega_{h k}\right]_{|\mathcal{H}| \times|\mathcal{K}|}$ denotes the subchannel assignment matrix. To solve (11), the elements of $\boldsymbol{\Omega}$ need to be mapped to the design variables of the solution vector in the ACO algorithm. We employ a mapping approach as shown in Fig. 3 where the first row of $\Omega$ forms the first $|\mathcal{K}|$ design variables of the solution vector in the ACO algorithm, the second row forms the second $|\mathcal{K}|$ design variables and so on. After mapping the subchannel assignment matrix to the solution vector, the problem in (11) can readily be solved using the ACO algorithm, as described below.

Step 1: Create $N$ ants. Initialize the solution vector, $\mathbf{x}_{n}=\left[x_{1}, \ldots, x_{i}, \ldots, x_{|\mathcal{H} \| \mathcal{K}|}\right]$ for all ants $n=1,2, \ldots, N$. Create a set of possible values for each design variable where each element in the set represents a node. Since the design variables in (11) are binary-valued, hence there are only two nodes in each layer, i.e., $j \in \mathcal{N}_{i}^{(n)}=\{1,2\}$ where the values of the first and second nodes are zero and one respectively. Set the iteration number, $t=1$. Assume equal amounts of pheromones for all the paths when $t=1$, by setting $\tau_{i j}(1)=1$ for all $i=1,2, \ldots,|\mathcal{H}||\mathcal{K}|$ and $j=1,2$. 
Step 2: Calculate the probability of ant $n$ selecting the path to node $j$ in layer $i, p_{i j}^{(n)}$ for all $n=1,2, \ldots, N, i=1,2, \ldots,|\mathcal{H}||\mathcal{K}|$ and $j=1,2 \operatorname{using}(14)$.

Step 3: Generate a random value $r_{n}$ in the range $(0,1)$ for all $n=1,2, \ldots, N$. Compare $r_{n}$ with for $i=1$. If $r_{n}$ is smaller than $p_{i j}^{(n)}, x_{1}$ is set to zero, otherwise $x_{1}$ is set to one for ant $n^{1}$. Repeat this for $i=2,3, \ldots,|\mathcal{H}||\mathcal{K}|$.

Step 4: Map the solution vector $x_{n}$ for $n=1$ back to the form of the subchannel assignment matrix. Evaluate the subchannel assignment matrix using the objective function in (11). Repeat this for $n=2,3, \ldots, N$.

Step 5: Determine the paths with the best and worst objective function values respectively using the following equations

$$
\begin{aligned}
& f_{\text {best }}=\max \left\{f^{(n)}\right\} \\
& f_{\text {best }}=\min \left\{f^{(n)}\right\}
\end{aligned}
$$

Step 6: Terminate the algorithm if the solution does not improve after $t_{\mathrm{NI}}$ iterations. Otherwise, all the ants are assumed to return to the home node and restart their food search. Update the iteration number $t=2$. Update the pheromone trail on each path using (15) and (16).

Step 7: Repeat Steps 2-6 until the algorithm does not improve after $t_{\mathrm{NI}}$ iterations.

To cope with the time varying channel conditions, the HMS executes the proposed ACO-based resource allocation (ACO-RA) algorithm once every period $T_{\mathrm{RA}}$. When executing the ACO-RA scheme, the HMS will prevent all the HeNBs in the network from transmitting data and only reference signals are allowed to be transmitted over the entire bandwidth for one TTI duration. Then, each HeNB identifies its interfering neighbors and estimates its resource demand before sending this information to the HMS.

It is noteworthy that the ACO-RA scheme only allocates resources to the HeNBs. Resource allocation among the FUEs served is made based on the scheduling policy implemented in each HeNB. In this study, the conventional proportional fair scheduling policy is employed.

\footnotetext{
1 It is noteworthy that path selection in ACO is determined using roulette-wheel selection, i.e., by matching the random number generated with the cumulative probability ranges associated with different paths based on the probabilities calculated using (14). As the design variables in our formulated problem are binary-valued, Step 3 only needs to compare one of the probabilities calculated with the generated random number without using roulette-wheel selection.
} 


\section{Performance Evaluation}

In this section, we evaluate and compare the performance of the ACO-RA scheme with two existing prominent schemes, namely the DRA [4] and C-DFP [5] schemes. We first describe the performance metrics used in our evaluation before presenting the simulation results and discussion.

\subsection{Performance Metrics}

The following performance metrics will be used to evaluate the performance of the ACO-RA scheme for LTE/LTE-A femtocell networks in terms of resource utilization, throughput satisfaction, fairness and throughput.

Resource Utilization: To assess the overall resource utilization, we use the objective function in (6). A large value of (6) indicates a higher resource utilization efficiency.

Throughput Satisfaction Ratio: Throughput satisfaction ratio (TSR) is a QoS metric introduced in [12], which is defined as $\frac{\sum_{k \in \mathcal{K}} \omega_{h k}}{D_{h}}$ where $\operatorname{TSR}(h)$ is the TSR of HeNB $h$. When $\operatorname{TSR}(h)=1$, the minimum resource demand $D_{h}$ is met. If $\operatorname{TSR}(h)>1$, it implies that HeNB $h$ is allocated an amount of resources exceeding $D_{h}$. The overall TSR can be calculated as the average of TSRs for all $h \in \mathcal{H}$, i.e., $\operatorname{TSR}=\frac{\sum_{h \in \mathcal{H}} \operatorname{TSR}(h)}{|\mathcal{H}|}$.

Fairness: Jain's fairness index [26] is employed to evaluate global fairness, which is defined as $\frac{\left(\sum_{h \in \mathcal{H}} \operatorname{TSR}(h)\right)^{2}}{|\mathcal{H}| \sum_{h \in \mathcal{H}}(\operatorname{TSR}(h))^{2}}$.

Throughput: The throughput achieved by an LTE/LTE-A femtocell network is defined as the number of bits successfully received by all the FUEs over a given duration. In our performance evaluation, the throughputs for different traffic classes are evaluated separately.

\subsection{Simulation Setting}

The performance of the ACO-RA scheme is evaluated using LTE-Sim [27,28]. In the simulation setup, a two-dimensional building of the $5 \times 5$ apartment grid type [29] is considered. Each apartment in the building has an area of $10 \times 10 \mathrm{~m}^{2}$ and accommodates a femtocell. The HeNBs in the building are connected to an HMS which performs resource allocation for all the HeNBs. We assume that a split spectrum approach is used and the femtocell network is allocated a bandwidth of $5 \mathrm{MHz}$, which is interference-free from the macrocell. We assume that each FUE receives a video flow, a voice over Internet Protocol 
Table 1 Simulation parameters of the femtocell network under study

\begin{tabular}{ll}
\hline Parameter & Setting \\
\hline Frame Structure & Frequency division duplexing (FDD) \\
Bandwidth & $5 \mathrm{MHz}(25$ Subchannels) \\
Simulated Duration & $60 \mathrm{~s}$ \\
Traffic Model & Video, VoIP and best-effort \\
Maximum Delay Allowable for GBR Flows & $0.1 \mathrm{~s}$ \\
HeNB Power Transmission & $20 \mathrm{dBm}$ (equally distributed among \\
& subchannels allocated) \\
Scheduler & Proportional fair \\
Building Type & $5 \times 5$ grid \\
Number of Buildings & 1 \\
Number of Apartments in a Building & 25 \\
Apartment Size & $10 \times 10$ m ${ }^{2}$ \\
Path Loss Model & Urban indoor channel model (Winner) [28] \\
Channel Fading Model & Rayleigh \\
Shadowing & Lognormal distribution with a zero mean \\
Femtocell Access Mode & and a standard deviation of 8 dB \\
\hline
\end{tabular}

(VoIP) flow and a best-effort flow. The minimum bit rates of the video and VoIP flows are set to $128 \mathrm{kbps}$ and $8 \mathrm{kbps}$ respectively. For the best-effort flow, an infinite buffer model is used. Therefore, the resource demand of each data flow, FUE and HeNB can then be estimated using (1), (2) and (3), respectively. The transmission power of each HeNB is set to $20 \mathrm{dBm}$ and it is distributed equally among all the subchannels allocated. A conventional proportional fair scheduler is employed in each HeNB. Other network parameters are listed in Table 1.

For the ACO-RA scheme, the preference vector $(x, y, z)$ is set to $(0.1,0.1$, 0.8 ) which is experimentally found to provide good network performance. For the ACO algorithm, the parameter settings are as follows: $\alpha=1, \zeta=1$, $\rho=0.1, \tau_{i j}(t=1)=1$ for all $i$ and $j, N=50$ and $t_{\mathrm{NI}}=20$. Periodic execution of the ACO-RA scheme at the HMS is set with $T_{\mathrm{RA}}=1 \mathrm{~s}$. We also assume that $T h=12 \mathrm{~dB}$ as in [20] for identifying the interfering HeNBs.

In our performance studies, we consider two scenarios: non-handover and handover scenarios. In the non-handover scenario, different numbers of FUEs per HeNB are set for the femtocell network and these FUEs will only stay within the coverage of their serving HeNBs; handover is not enabled. Based on the aforementioned scenario, we evaluate the performance of the LTE/LTE-A femtocell network with different activity ratios, $r$ [29], which define the probabilities of HeNBs in activity within the building. An example of an LTE/LTE-A femtocell network with $r=0.2,0.4,0.6,0.8$ and 1 is illustrated in Fig. 4 . In the non-handover scenario, we evaluate the femtocell network with $r=0.2$ and $r=0.6$ to study the effect of femtocell density to the network performance. In the handover scenario, a fixed number of HeNBs are randomly activated within the apartment and different total numbers of handover-enabled FUEs are set for the femtocell network. In this scenario, we set the number of HeNBs 


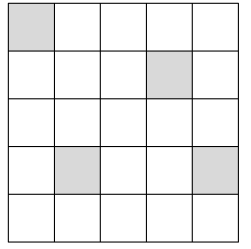

$r=0.2$

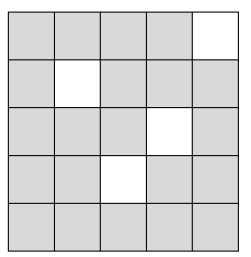

$r=0.8$

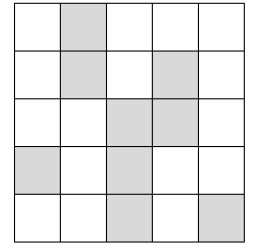

$r=0.4$

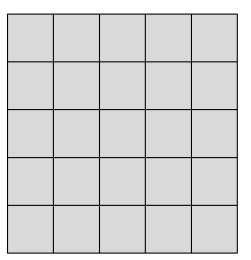

$r=1$

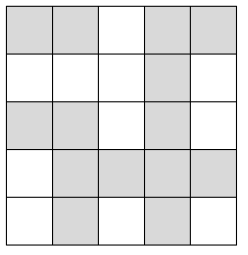

$r=0.6$

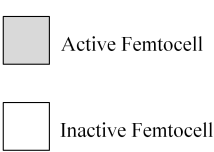

Fig. 4 LTE/LTE-A femtocell network with $r=0.2,0.4,0.6,0.8$ and 1

to 15 and a handover scheme based on signal strengths is used. The movement of each FUE is based on a random direction model in [27] whereby each FUE randomly chooses a direction and travels until the simulation boundary is reached and a new direction is then chosen. The speed of each FUE is set to $3 \mathrm{~km} / \mathrm{h}$. All results obtained are averaged over 20 simulation runs.

\subsection{Results and Discussion for the Non-Handover Scenario}

The overall resource utilization achieved by all the schemes is presented in Fig. 5. When $r=0.2$, the C-DFP scheme exhibits increasing resource utilization with the number of FUEs per HeNB but its resource utilization declines when the number of FUEs per HeNB is four. This is because the resource demand of some HeNBs exceeds the amount of available resources, resulting in failure of the C-DFP scheme in assigning resources to these HeNBs. Unlike the C-DFP scheme, the ACO-RA scheme shows slightly declining resource utilization when $r=0.2$ because it reduces the number of resources allocated to each HeNB in order to reduce the likelihood of multiple interfering HeNBs sharing the same resources. In the same scenario, the DRA scheme always attains the lowest resource utilization due to its resource allocation mechanism that does not maximize resource utilization. When $r=0.2$, the ACO-RA scheme and the DRA scheme initially achieves higher resource utilization than the C-DFP scheme because of the low interference in this scenario, thus allowing the former two schemes to maximize resource utilization to a higher extent. However, the ACO-RA scheme and the DRA scheme reduce their resource utilization due to the high interference encountered in scenarios with larger numbers of FUEs per HeNB, thus resulting in lower resource utilization than the C-DFP scheme. When $r=0.6$, the high interference causes the ACO-RA scheme and 


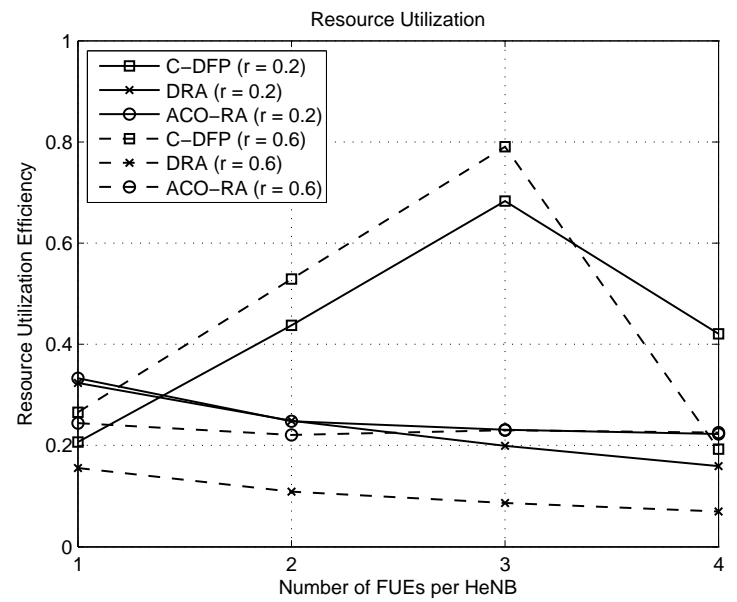

Fig. 5 Overall resource utilization for the non-handover scenario

the DRA scheme to further reduce their resource utilization. Meanwhile, the C-DFP scheme increases its resource utilization to fulfill the higher resource demands in this scenario due to the higher interference. However, it is again unable to assign resources to the HeNBs at four FUEs per HeNB because these HeNBs have resource demands exceeding the amount of available resources.

The average TSR performance achieved by all the schemes is illustrated in Fig. 6. When $r=0.2$, the ACO-RA scheme initially achieves a good TSR performance but the performance declines with the increase in the number of FUEs per HeNB due to the increasing interference that causes the ACO-RA scheme to reduce the amount of resources allocated to each HeNB. Meanwhile, the DRA scheme demonstrates a similar performance trend as that of the ACO-RA scheme. When $r=0.6$, the average TSR performance of both the ACO-RA scheme and the DRA scheme deteriorates because the interference in this scenario is higher. In both scenarios with $r=0.2$ and $r=0.6$, the CDFP scheme consistently maintains a unity TSR but the TSR declines when the number of FUEs per HeNB is four because it fails to assign resources to the HeNBs that have resource demands exceeding the amount of available resources.

Fig. 7 shows the global fairness performance of the three schemes. The ACO-RA scheme maintains a relatively high level of global fairness at around 0.8 in both scenarios with $r=0.2$ and $r=0.6$. The global fairness performance of the DRA scheme is inferior to the other two schemes, especially when $r=$ 0.6. This is because the DRA scheme does not provide fair amounts of resources to the HeNBs to satisfy their resource demands. The C-DFP scheme achieves the highest fairness index because it always achieves a unity TSR value for each HeNB but the high fairness index declines to the lowest at four FUEs per HeNB due to its inability to assign resources to HeNBs with resource demands exceeding the amount of available resources. 


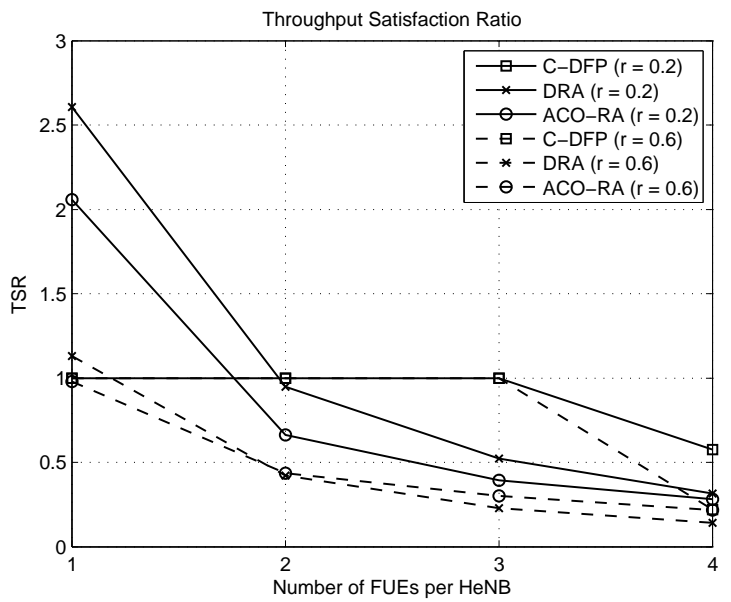

Fig. 6 Average TSR performance for the non-handover scenario

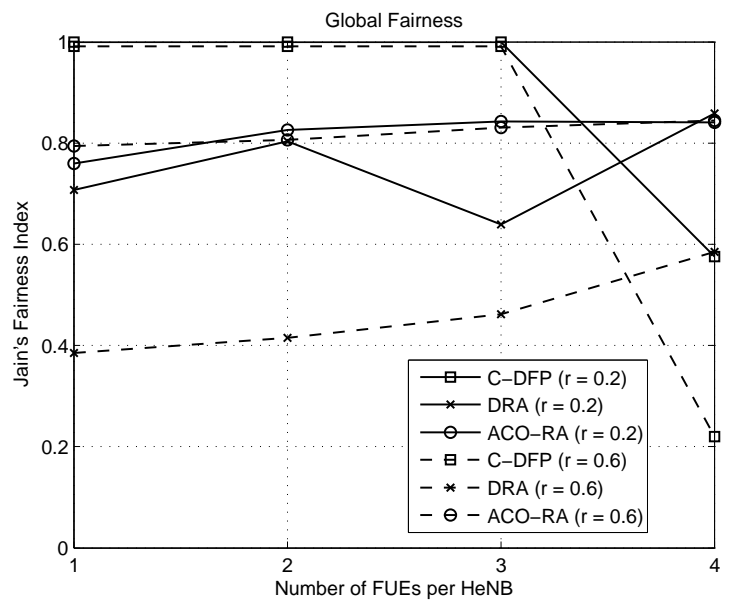

Fig. 7 Global fairness performance for the non-handover scenario

In Fig. 8, when $r=0.2$, the ACO-RA scheme shows the best overall throughput performance for video, VoIP and best-effort flows overall due to its inherent resource allocation feature that minimizes interference. However, it does not outperform the other two schemes when $r=0.6$ because it reduces the amount of resources allocated to each HeNB in order to reduce the high interference in this scenario while guaranteeing high global fairness. Nevertheless, the ACO-RA scheme performs satisfactorily in this scenario. When $r=0.6$, the DRA scheme demonstrates high video and VoIP throughput performance, as shown in Fig. 8a and 8b, respectively, because it fully eliminates interference by avoiding resource overlaps between interfering HeNBs. However, the 


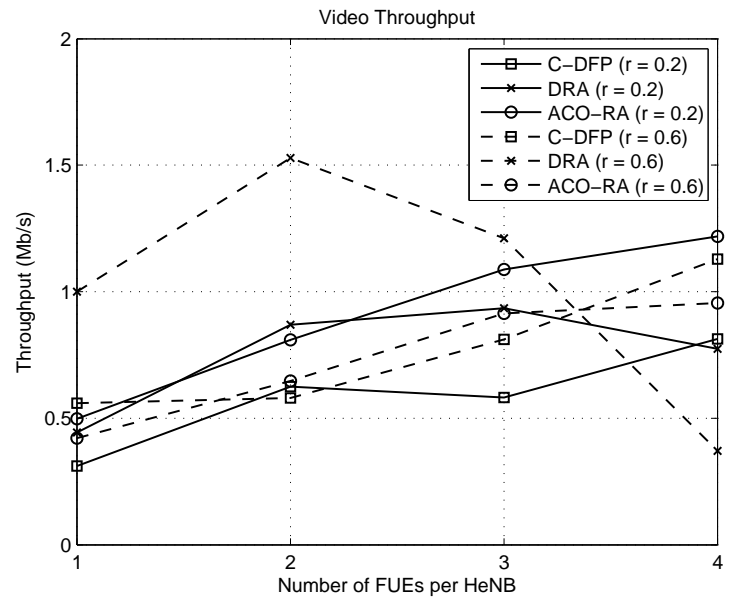

(a)

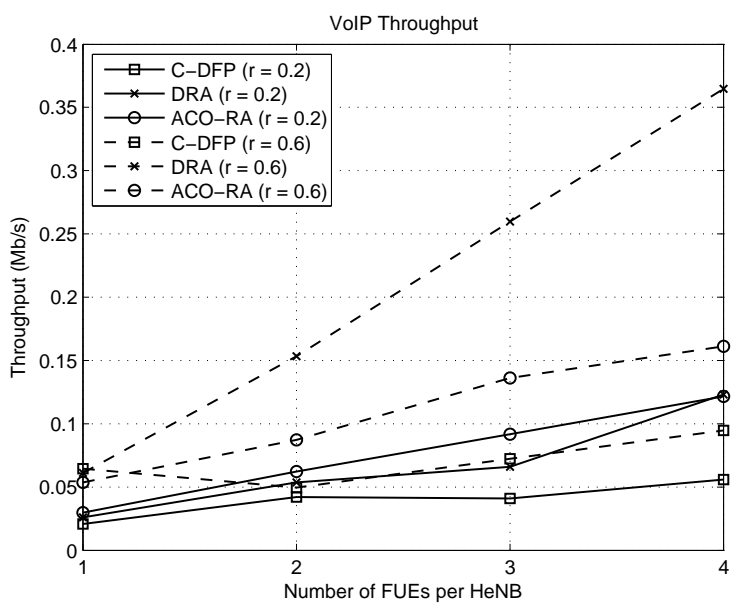

(b)

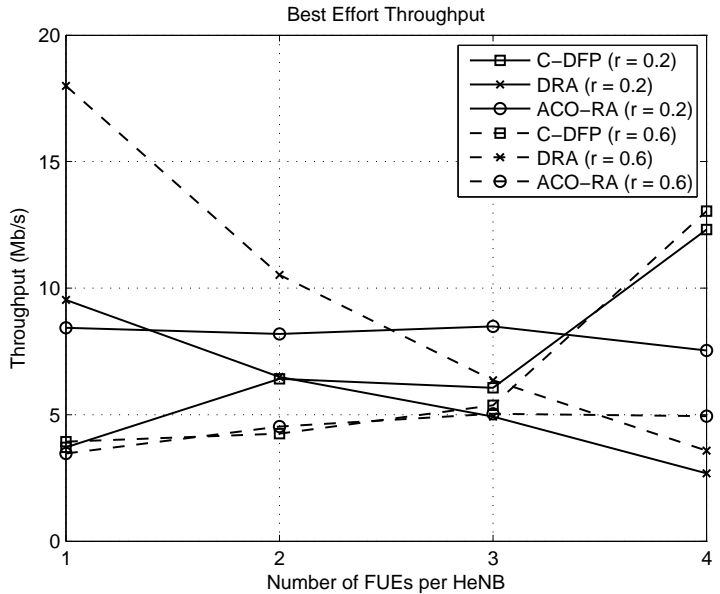

(c)

Fig. 8 Throughput performance of (a) video flows, (b) VoIP flows and (c) best-effort flows for the non-handover scenario. 
video throughput performance declines at four FUEs per HeNB when $r=0.6$ because the HeNBs do not receive sufficient resources, as indicated by their inferior TSR performance shown in Fig. 6. Besides that, the DRA scheme only provides more resources to the HeNBs which are less being interfered with, thus resulting in higher throughput but lower global fairness, as shown in Fig. 7. A similar performance trend of the DRA scheme for best effort flows is observed in Fig. ]reffig8c. The C-DFP scheme does not perform well in both scenarios when $r=0.2$ and $r=0.6$ due to its inefficient interference mitigation strategy and inability to assign resources when the available resources are insufficient to meet the resource demands of the HeNBs.

It is noteworthy that, overall, the ACO-RA scheme demonstrates better performance when resource utilization, global fairness and interference mitigation are jointly considered. It can be observed that, unlike the ACO-RA scheme which exhibits good performance in all these aspects, the C-DFP scheme does not perform well in terms of throughput whereas the DRA scheme is inferior in terms of resource utilization and global fairness.

\subsection{Results and Discussion for the Handover Scenario}

Fig. 9 shows the resource utilization performance of the three schemes in the handover scenario. It can be seen that the C-DFP scheme attains higher resource utilization than the other two schemes but becomes inferior to the ACO-RA scheme when the total number of FUEs is 80. This is because the former is unable to assign resources to the HeNBs that demand more than the available resources. The ACO-RA scheme maintains moderate resource utilization performance while the DRA scheme attains the lowest resource utilization performance. The performance trend in Fig. 9 is similar to that in Fig. 5 because all the schemes regularly update their resource allocation.

In Fig. 10, the C-DFP scheme achieves the highest average TSR performance but becomes slightly inferior to the ACO-RA scheme at 60 FUEs due to the former's inability to assign resources to the HeNBs that request more than the available resources. Both the ACO-RA scheme and the DRA scheme attain low TSR performance with the performance of the latter being lower due to the fact that the interference is high when the total number of FUEs is large, thereby resulting in less resources being allocated to each HeNB. The TSR performance of the DRA scheme is slightly more vulnerable in the handover scenario and declines slightly because it does not fulfill the resource demand of each HeNB and thus does not take into account the resource demand of handover FUEs.

Fig. 11 depicts a similar performance trend as in Fig. 7 where the ACORA scheme maintains a considerably high fairness index; the C-DFP scheme achieves the highest fairness index but becomes inferior to the other two schemes at 60 FUEs; the DRA scheme attains the lowest fairness performance overall. 


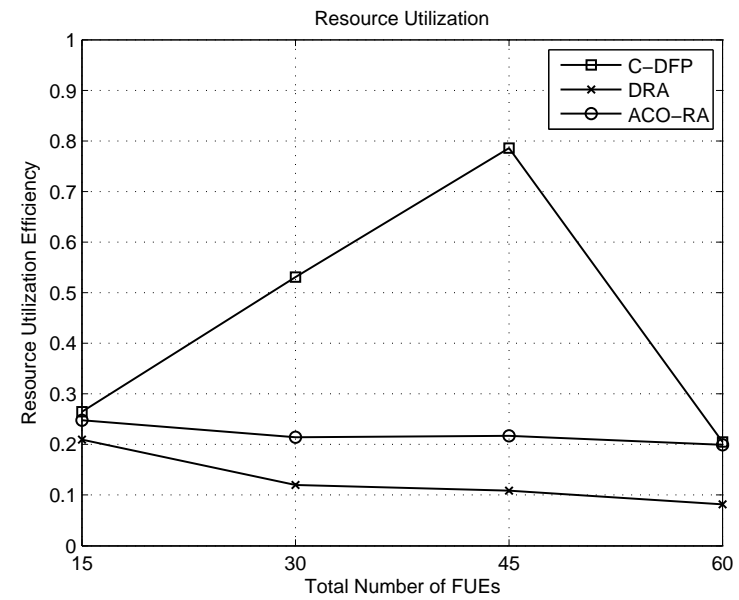

Fig. 9 Overall resource utilization for the handover scenario

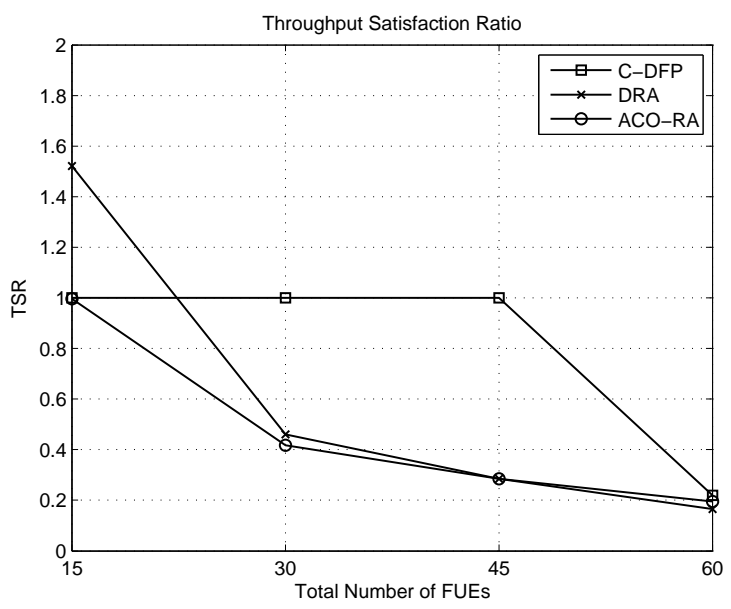

Fig. 10 Average TSR performance for the handover scenario

Fig. 12 shows the throughput performance of the three schemes for video, VoIP and best-effort flows. It can be observed that the throughput performance of the three schemes in the handover scenario is inferior to that in the nonhandover scenario. This is because the handover process results in throughput degradation since data transmission is halted during the handover process. The DRA scheme is most vulnerable to the handover process because it does not fulfill the resource demand of each HeNB and therefore some HeNBs suffer from resource starvation. The ACO-RA scheme is also affected by the handover scenario but it nevertheless performs better than the DRA scheme because it aims to satisfy the resource demand of each HeNB and regularly updates its 


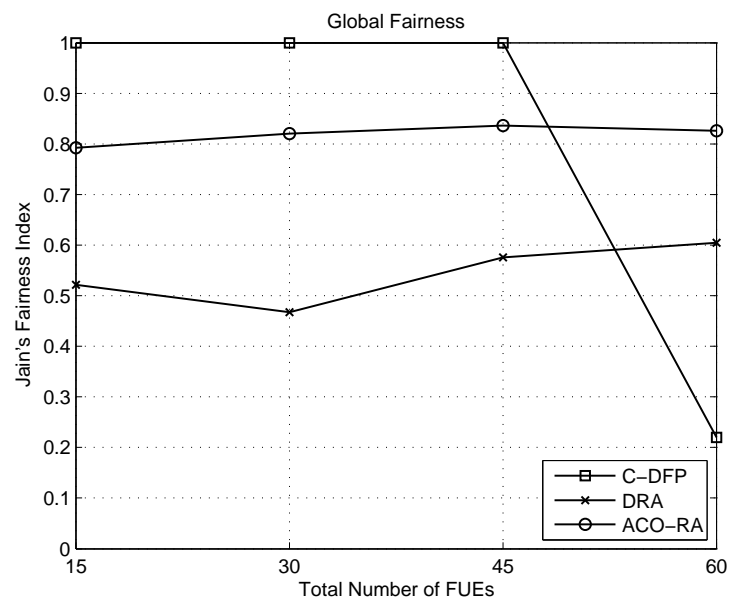

Fig. 11 Global fairness performance for the handover scenario

resource allocation. The C-DFP scheme also regularly updates its resource allocation but it is unable to assign resources to the HeNBs that demand excessive amounts of resources.

\subsection{Complexity Analysis}

This section analyzes the complexity of the three schemes. In the C-DFP scheme, the resource broker requires a maximum of $|\mathcal{H}|^{2}|\mathcal{K}|^{2}$ operations to complete resource allocation among all the HeNBs. Therefore, the C-DFP scheme has an asymptotic complexity of $\mathcal{O}\left(|\mathcal{H}|^{2}|\mathcal{K}|^{2}\right)$. The DRA scheme requires $\frac{(10|\mathcal{H}||\mathcal{K}|)}{I}$ operations to complete the whole resource allocation process where $I$ is the number of interfering HeNBs. Therefore, the asymptotic complexity of the DRA scheme is of $\mathcal{O}(|\mathcal{H}||\mathcal{K}|)$. On the other hand, the ACORA scheme requires $\left(t_{\max }+t_{\mathrm{NI}}\right)$ iterations and $N$ ants to find the solution where $t_{\max }$ is the number of iterations for the ACO-RA scheme to converge. Thus, the maximum number of operations required by the ACO-RA scheme is $N\left(t_{\max }+t_{\mathrm{NI}}\right)$ and the asymptotic complexity is of $\mathcal{O}\left(N t_{\max }\right)$.

To compare the complexity of the three schemes, we obtain the convergence performance of the ACO algorithm in a typical femtocell network scenario using the same parameter settings in Section 3.2 for 100 runs, as depicted in Fig. 13. It is observed that the ACO algorithm converges at 40, 70, 80, 90 and 100 iterations for 5, 10, 15, 20 and 25 femtocells respectively. A comparison of the maximum number of operations between all the schemes is shown in Table 2. The complexity of the ACO-RA scheme is comparable with that of the DRA scheme; with the latter being lower for 5, 10 and 15 femtocells and the former being lower for 20 and 25 femtocells. However, the C-DFP scheme always incurs a huge complexity for all numbers of femtocells. 


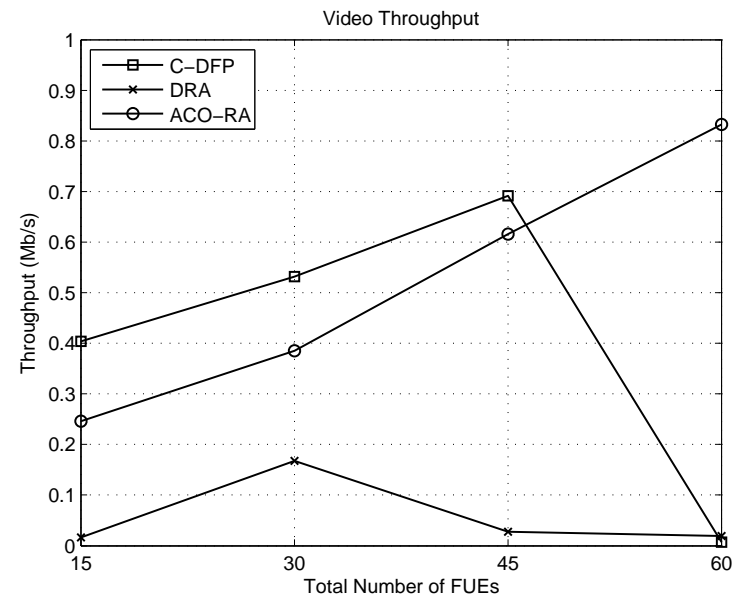

(a)

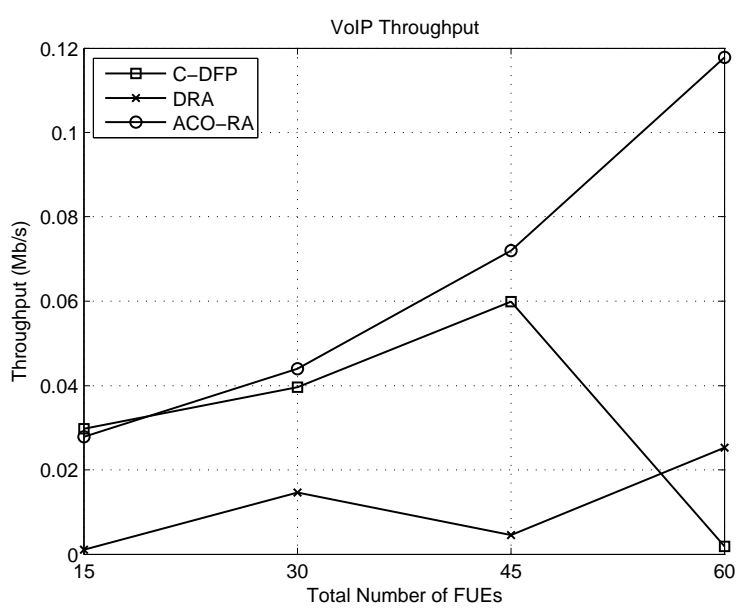

(b)

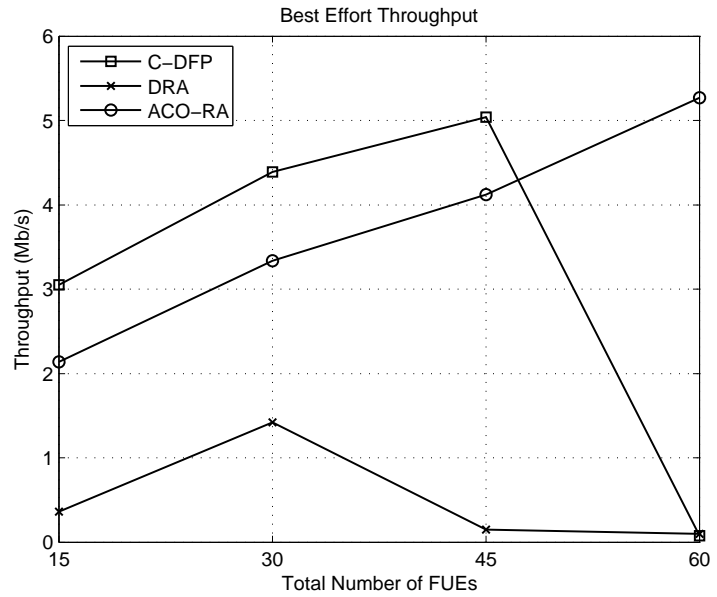

(c)

Fig. 12 Throughput performance of (a) video flows, (b) VoIP flows and (c) best-effort flows for the handover scenario. 


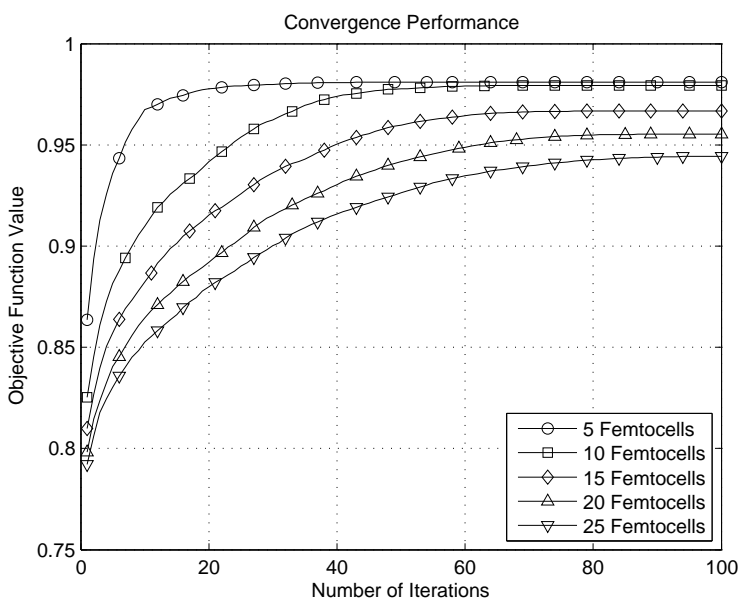

Fig. 13 Average convergence performance of the ACO algorithm for different numbers of femtocells

Table 2 Complexity comparison between resource allocation schemes

\begin{tabular}{llllll}
\hline \multirow{2}{*}{ Scheme } & \multicolumn{5}{c}{ Maximum Number of Operations } \\
\cline { 2 - 6 } & 5 Femtocells & 10 Femtocells & 15 Femtocells & 20 Femtocells & 25 Femtocells \\
\hline C-DFP & 15625 & 62500 & 140625 & 250000 & 390625 \\
DRA & 1250 & 2500 & 3750 & 5000 & 6250 \\
ACO-RA & 3000 & 4500 & 5000 & 5500 & 6000 \\
\hline
\end{tabular}

\section{Conclusion}

This paper has introduced a new multi-objective resource allocation scheme for LTE/LTE-A femtocell networks that aims to attain minimum co-tier interference with high resource utilization and global fairness. In this scheme, three objective functions are formulated to respectively maximize resource utilization efficiency, guarantee a high degree of fairness and minimize co-tier interference. These objective functions are merged using a weighted sum approach to form a single multi-objective optimization problem. A proper weight setting method is also proposed to equalize the smallest value of the three objective functions. To solve the optimization problem, an ACO algorithm is employed and adapted to find the Pareto-optimal solution. Simulation results have demonstrated that the proposed ACO-RA scheme performs jointly relatively well in all aspects of resource utilization, global fairness and interference mitigation. More importantly, it continues to exhibit good performance even in the handover scenario. The complexity of the proposed scheme is shown to be significantly lower than that of the C-DFP scheme and is comparable to that of the DRA scheme, thus making it an attractive and efficient resource allocation scheme suitable for self-organizing LTE/LTE-A networks. 
Acknowledgements This work is supported in part by Yayasan Universiti Multimedia (YUM) foundation and the fundamental research grant scheme (FRGS), Ministry of Higher Education, Malaysia.

\section{References}

1. Saquib, N., Hossain, E., Le, L. B., \& Kim, D. I. (2012). Interference management in OFDMA femtocell networks: Issues and approaches. IEEE Wireless Communications, $19(3), 86-95$.

2. Chandrasekhar, V., \& Andrews, J. (2009). Spectrum allocation in tiered cellular networks. IEEE Transactions on Communications, 57(10), 3059 - 3068.

3. Rahman, M., \& Yanikomeroglu, H. (2010). Enhancing cell-edge performance: A downlink dynamic interference avoidance scheme with inter-cell coordination. IEEE Transactions on Wireless Communications, 9(4), 1414 - 1425.

4. Sundaresan, K., \& Rangarajan, S. (2009) Efficient resource management in OFDMA femtocells. In: Proceedings of the ACM International Symposium on Mobile Ad Hoc Networking and Computing (MobiHoc) (pp. 3342). New Orleans, Louisiana, USA.

5. Lopez-Perez, D., Valcarce, A., de la Roche, G., \& Zhang, J. (2009). OFDMA femtocells: A roadmap on interference avoidance. IEEE Communications Magazine, 47(9), 4148.

6. Saha, R. K. (2013). Modified proportional fair scheduling for resource reuse and interference coordination in two-tier LTE-advanced systems. International Journal of Digital Information and Wireless Communications, 3(2), $9-28$.

7. Liang, Y.-S., Chung, W.-H., Ni, G.-K., Chen, I.-Y., Zhang, H., \& Kuo, S.-Y. (2012). Resource allocation with interference avoidance in OFDMA femtocell networks. IEEE Transactions on Vehicular Technology. 61(5), 22432255.

8. Lee, K., Jo, O., \& Cho, D.-H. (2011). Cooperative resource allocation for guaranteeing intercell fairness in femtocell networks. IEEE Communications Letters, 15(2), 214216.

9. Lopez-Perez, D., Chu, X., Vasilakos, A. V., \& Claussen, H. (2013) Power minimization based resource allocation for interference mitigation in OFDMA femtocell networks. IEEE Journal on Selected Areas in Communications, 32(12), 333 - 344.

10. Zheng, K., Wang, Y., Lin, C., Shen, X., \& Wang, J. (2011). Graph-based interference coordination scheme in orthogonal frequency-division multiplexing access femtocell networks. IET Communications, 5(17), 2533-2541.

11. Kim, B.-G., Kwon, J.-A., \& Lee, J.-W. (2013). Subchannel allocation for the OFDMAbased femtocell system, Computer Networks, 57(17), 3617 - 3629.

12. Hatoum, A., Langar, R., Aitsaadi, N., Boutaba, R., \& Pujolle, G. (2013). Clusterbased resource management in OFDMA femtocell networks with QoS guarantees. IEEE Transactions on Vehicular Technology, 63(5), 2378 - 2391.

13. Lee, Y. L., Chuah, T. C., Loo, J., \& Vinel, A. (2014). Recent advances in radio resource management for heterogeneous LTE/LTE-A networks. IEEE Communications Surveys and Tutorials, $16(4), 2142-2180$.

14. Jo, H.-S., Xia, P., \& Andrews, J. G. (2011). Downlink femtocell networks: Open or closed?. IEEE International Conference on Communications (ICC) (pp. 1 - 5). Kyoto, Japan.

15. Yun, S., Yi, Y., Cho, D.-H., \& Mo, J. (2011). Open or close: On the sharing of femtocells. In: Proceedings of the IEEE INFOCOM (pp. 116 - 120). Shanghai, Japan.

16. Evolved Universal Terestrial Radio Access (E-UTRA) and Evolved Universal Terestrial Radio Access Network (E-UTRAN), Overall description: Stage 2, TS 36.300, Dec. 2013.

17. Technical Specification Group Radio Access Network; Evolved Universal Terrestrial Radio Access (E-UTRA); Physical Channels and Modulation, TS 36.211, Sep. 2012.

18. Pedersen, K. I., Kolding, T. E., Frederiksen, F., Kovacs, I. Z., Laselva, D., \& Mogensen, P. E. (2009). An overview of downlink radio resource management for UTRAN Long-Term Evolution. IEEE Communications Magazine. 47(7), 86 - 93.

19. Capozzi, F., Piro, G., Grieco, L. A., Boggia, G., \& Camarda, P. (2013). Downlink packet scheduling in LTE cellular networks: Key design issues and a survey. IEEE Communications Surveys and Tutorials. 15(2), $678-700$. 
20. Lopez-Perez, D., Chu, X., \& Zhang, J. (2012). Dynamic downlink frequency and power allocation in OFDMA cellular networks. IEEE Transactions on Communications. 60(10), 2904-2914.

21. Pareto, V., \& Schwier, A. S. (1971). Manual of Political Economy. A.M. Kelley.

22. Marler, R. T., \& Arora, J. S. (2004). Survey of multi-objective optimization methods for engineering. Structural and Multidisciplinary Optimization, 26(6), 369-395.

23. Zadeh, L. A. (1963). Optimality and non-scalar-valued performance criteria. IEEE Transactions on Automatic Control, 8(1), 5960.

24. Dorigo, M., Maniezzo, V., \& Colorni, A. (1996). Ant system: Optimization by a colony of cooperating agents. IEEE Transactions on Systems, Man, and Cybernetics, Part B: Cybernetics, 26(1), 29-41.

25. Rao, S. S. (2009). Engineering Optimization: Theory and Practice. John Wiley \& Sons.

26. Jain, R. (1991). The Art of Computer Systems Performance Analysis. John Wiley \& Sons.

27. Piro, G., Grieco, L. A., Boggia, G., Capozzi, F., \& Camarda, P. (2011). Simulating LTE cellular systems: An open-source framework. IEEE Transactions on Vehicular Technology, $60(2), 498-513$.

28. Capozzi, F., Piro, G., Grieco, L. A., Boggia, G., \& Camarda, P. (2012). On accurate simulations of LTE femtocells using an open source simulator. EURASIP Journal on Wireless Communications and Networking, 2012(328), 1- 13.

29. Simulation Assumptions and Parameters for FDD HeNB RF Requirements. R4-092042. 